\title{
Histopathological Features and Clinical Variants of Biopsy Confirmed Psoriasis Cases in a Tertiary Care Setting in Kerala
}

\author{
Anjukrishna Sasikala Appukkuttan¹, Retheesh Kollerazhikathu Haridasan², Betsy Annamma Jose ${ }^{3}$ \\ ${ }^{1}$ Department of Pathology, Sree Gokulam Medical College, Thiruvananthapuram, Kerala, India. ${ }^{2}$ Department \\ of Community Medicine, Government Medical College, Thiruvananthapuram, Kerala, India. ${ }^{3}$ Department of \\ Community Medicine, Government Medical College, Thiruvananthapuram, Kerala, India.
}

\section{ABSTRACT}

\section{BACKGROUND}

Psoriasis is a chronic, immune mediated, relapsing, papulosquamous disease having a high prevalence. Since it affects other organ systems such as musculoskeletal system, gastrointestinal system and the eye, it can lead to considerable disability. Although only rarely life threatening, it has high morbidity due to its chronicity and absence of cure.

\section{METHODS}

This study was conducted over a period of 2 years in the Department of Pathology, Medical College, Thiruvananthapuram. A total of 217 skin biopsy specimens in which a clinical diagnosis / differential diagnosis of psoriasis was made, was studied during this period.

\section{RESULTS}

108 cases out of 217 which were histopathologically diagnosed as psoriasis were studied in detail. Male predominance was noted in the study population. The mean duration of disease in this study was 6.69 yrs. Fifty percentage of the patients had associated comorbidities with hypertension outnumbering others. Among male patients, 26 (32.5\%) had the habit of smoking. The most common presentation was as erythematous scaly plaques, with pruritus being the second most common presentation. Histopathology proved to be conclusive of psoriasis in all cases. Hyperkeratosis was seen in all cases which was the most consistent histopathological feature. Confluent parakeratosis which is one of the characteristic features of psoriasis was seen in 62 (57.4\%) cases with the rest being focal. Other epidermal features studied were papillomatosis, hypogranulosis, suprapapillary thinning, and basal mitotic figures. Spongiosis was seen in 83 (76.9\%), exocytosis of neutrophils in 66 (61.1\%) and Munro's micro abscess in 42 (38\%) cases. Dilated blood vessel was the most common dermal change observed, seen in 105 (97 \%). Lymphocytes were the most frequent upper dermal inflammatory infiltrate observed. Oedema was seen in $5(4.6 \%)$ of cases.

\section{CONCLUSIONS}

Psoriasiform lesions pose diagnostic dilemma to the treating clinician. To provide a clear-cut diagnosis, histopathological evaluation is essential. It is also important to differentiate between the different variants of psoriasis in the context of treatment. It has an important role in the follow up of psoriatic patients.

\section{KEY WORDS}

Psoriasis, Papulosquamous, Erythematous Scaly Plaques, Confluent Parakeratosis, Regular Acanthosis, Hyperkeratosis
Corresponding Author: Dr. Retheesh Kollerazhikathu Haridasan, Department of Community Medicine, Government Medical College, Thiruvananthapuram, Kerala, India. E-mail:drretheeshkh@gmail.com

DOI: $10.14260 /$ jemds/2020/514

How to Cite This Article: Appukkuttan AS, Haridasan RK, Jose BA. Histopathological features and clinical variants of biopsy confirmed psoriasis cases in a tertiary care setting in Kerala. J Evolution Med Dent Sci 2020;9(33):23722376, DOI: 10.14260/jemds/2020/514

Submission 18-11-2019,

Peer Review 07-07-2020,

Acceptance 15-07-2020,

Published 17-08-2020.

Copyright (C) 2020 JEMDS. This is an open access article distributed under Creative Commons Attribution License [Attribution 4.0 International (CC BY 4.0)] 


\section{BACKGROUND}

Psoriasis is a chronic immune mediated lifelong disease that primarily affects the skin. Since it affects other organ systems such as musculoskeletal system, gastrointestinal system and the eye, it can lead to considerable disability. Although only rarely life threatening, it has high morbidity due to its chronicity and absence of cure. It affects the quality of life as patients are embarrassed by their appearance, have reduced levels of employment and income. Psoriasis is a relapsing papulosquamous dermatitis characterized by hyper proliferation of epidermis. It is a multifactorial disease with a genetic background ${ }^{1}$. It comprises of well circumscribed red scaly papules and plaques ${ }^{2}$. The disease has a major impact on the health care systems and on society in general because of its high prevalence. Due to these implications, the clinician and pathologist need to work in close collaboration to offer a diagnosis of psoriasis and to differentiate between other papulosquamous lesions. Psoriasis has many different clinical variants which mimic various dermatological conditions like secondary syphilis, seborrheic dermatitis, pityriasis rosea and parapsoriasis. The recurring nature and prognosis of psoriasis differs from other psoriasiform dermatitis. Clinical features alone are not reliable and can cause diagnostic dilemma. So, it is essential to have a histopathological confirmation of the clinical diagnosis for the satisfactory management of the conditions. Further, histological material provides evidence and can be preserved for future review. So as in other dermatological conditions, histopathology is considered as gold standard for diagnosis. 23,4 My study aims at evaluating the demographic profile, clinical features and clinico histopathological correlation. I also looked at the histopathological features useful in subtyping psoriasis.

\section{Objectives}

1. To assess the clinical and histopathological features of psoriasis

2. To study the clinico pathological correlation in patients with psoriasis

3. To identify the histopathological features useful in subtyping psoriasis.

\section{METHODS}

This is a case series study conducted at the Department of Dermatology OPD / IPD and Department of Pathology, Government Medical College, Thiruvananthapuram from 2011 January to 2012 December.

\section{Inclusion Criteria}

Skin biopsy specimens from all consecutive new cases received in the department with a clinical diagnosis/ differential diagnosis of psoriasis was included.

\section{Exclusion Criteria}

Those patients, who did not give consent.

\section{Method of Data Collection}

General information regarding the patient such as age, sex, age at onset, clinical presentation, duration, history of smoking and prior treatment, family history, comorbidities associated, and various histopathological parameters were studied. The skin biopsy specimens fixed in $10 \%$ formalin received in the Department of Pathology were taken. These tissues were processed and 5 micrometer thickness sections were taken from paraffin embedded tissues. The sections were stained with haematoxylin and eosin. Detailed study of histopathologically diagnosed cases of psoriasis was done. Histopathological features helpful in identifying different types of psoriasis were assessed. Results were correlated and compared with the clinical diagnosis.

\section{Sample Size and Sampling}

All cases fulfilling the inclusion criteria who attended Dermatology Department were included in the case series. Among the 217 cases, 108 which were histopathologically diagnosed as psoriasis were studied in detail. No sampling techniques were employed.

\section{Statistical Analysis}

Data entry and analysis were done using statistical software "Epi Info". Categorical variables were expressed as proportions and quantitative variables as mean and standard deviation

\section{RESULTS}

During the study period, out of the 217 cases in which there were a clinical/ differential diagnosis of psoriasis, 108 (49.8 $\%)$ cases were histopathologically diagnosed as psoriasis. The age ranged from 5 yrs. in the youngest to $80 \mathrm{yrs}$. in the oldest. Mean age of the patients was 44 years. The most common age group of patients in this study was between 31.25 and 55.75 years. The mean age among males was 44.2 yrs. and for that of females was 43.7 yrs. $25.9 \%$ belong to the age category of 5160. Majority of the patients were males $(74.1 \%)$ with a male: female ratio of 2.9:1 the mean duration of disease at histopathologic confirmation was 6.69 years. Most of the people ( $40.8 \%$ ) had a duration of disease between 2-9 years. Out of the 108 patients 47 (43.5\%) patients had undergone prior treatment before biopsy.

\begin{tabular}{|ccc|}
\hline Type of Lesion & Number & Percentage \\
Erythematous scaly plaques & 79 & 73 \\
Scalp scaling & 43 & 39.8 \\
Nail changes & 53 & 49 \\
Gen. Exfoliation & 18 & 16.6 \\
Pustules & 16 & 14.8 \\
Pruritus & 63 & 58.3 \\
Palm \& sole involvement & 23 & 21.3 \\
Arthritis & 5 & 4.6 \\
\hline \multicolumn{2}{c}{ Table 1. Clinical Features } \\
\hline
\end{tabular}

The commonest clinical features observed were Erythematous scaly plaques, pruritus and nail changes. Overall 26 (24.17) patients were smokers. But when the females were excluded (none gave history of smoking) the percentage rose to 32.5 percentage among males. Hypertension (22.9\%), diabetes 
mellitus (14.8\%), both HTN and DM (7.4\%) and Coronary artery disease (6.48\%) were the commonly associated co morbidities.

\begin{tabular}{|ccc|}
\hline Feature & $\begin{array}{c}\text { Number } \\
\mathbf{n = 1 0 8}\end{array}$ & $\begin{array}{c}\text { Percentage } \\
\text { (\%) }\end{array}$ \\
\hline Prominent dermal blood vessel & 105 & 97.3 \\
Lymphocyte as the upper dermal infiltrates & 70 & 64.8 \\
Normal Connective tissue \& Appendages & 99 & 91.7 \\
\hline \multicolumn{2}{|c|}{ Table 3. Histopathology - Dermis } \\
\hline
\end{tabular}

Dilated and tortuous blood vessels are one of the most characteristic features of psoriasis. Prominent blood vessels were noted in 105 (97.3\%) of the cases. Most commonly (64.8 $\%)$ observed dermal infiltrates were lymphocytes. 34 (31.5\%) infiltrates were both lymphocytes and neutrophils 99 (91.7\%) participants had normal connective tissue and appendages. Elastolysis and oedema of connective tissue and appendages were noted only in 4 (3.7\%) and 5 (4.6\%) respectively.

\begin{tabular}{|ccc|}
\hline Diagnosis & Number & Percentage \\
\hline Psoriasis vulgaris & 93 & 86.1 \\
Pustular psoriasis & 4 & 3.7 \\
Psoriasis with eczematisation & 4 & 3.7 \\
Psoriasis with postulation & 3 & 2.8 \\
Guttate psoriasis & 2 & 1.85 \\
Follicular psoriasis & 2 & 1.85 \\
Total & $\mathbf{1 0 8}$ & $\mathbf{1 0 0}$ \\
\hline Table 4. Different Variants Encountered in the Study \\
\hline
\end{tabular}

\section{Histopathology}

Parakeratosis is defined as retained nuclei in the stratum corneum layer. Parakeratosis was seen 106 (98.1\%) of cases. Confluent parakeratosis was observed in 62 (57.4\%) of cases. Acanthosis was observed in all cases. Elongated bulbous $(75 \%)$ rete ridges are a characteristic feature of psoriasis. Hyperkeratosis is defined as the thickening of the stratum, corneum. It was noted in all the cases. Neutrophil Exocytosis was observed in 66 (61.1\%) of cases. Munro Micro Abscess is defined as collection of neutrophils in the stratum corneum layer. It was seen in 41 (38\%) of the participants. Spongiosis is the presence of intraepidermal and intercellular oedema. It was noted in 83 (76.9\%) of the cases. Regular Acanthosis constituted $79(73.1 \%)$ of cases. Suprapapillary thinning is constituted $83(76.9 \%)$ of the cases. It is one of the defining features of psoriasis. Hypogranulosis is defined as the decrease in the granular layer. It was noted in 95 (87.9\%) of the cases. Kogoj abscess was observed in $6(5.6 \%)$ of the cases. It is the defined as the collection of neutrophils in the stratum spinosum layer. Bulbous rete ridges were noted in most of the cases $81(74.1 \%)$ of the cases. Increased mitotic figures are noted in the basal and spinous layers in psoriasis. It was noted in $80(74.1 \%)$ of the cases.

\begin{tabular}{|ccc|}
\hline Histopathologic Features & Present & Absent \\
\hline Hyperkeratosis & $108(100 \%)$ & $0(0 \%)$ \\
Orthokeratosis & $0(0 \%)$ & $108(100 \%)$ \\
Exocytosis of neutrophils & $66(61.1 \%)$ & $42(38.8 \%)$ \\
Muno's micro abscess & $41(38 \%)$ & $67(62 \%)$ \\
Spongiosis & $83(76.9 \%)$ & $25(23.1 \%)$ \\
Papillomatosis & $6(5.5 \%)$ & $102(94.4 \%)$ \\
Supra papillary thinning & $83(76.9 \%)$ & $25(23.1 \%)$ \\
Hypogranulosis & $95(87.9 \%)$ & $13(12.04 \%)$ \\
Kogoj abscess & $6(5.6 \%)$ & $102(94.4 \%)$ \\
Mitotic figures & $80(74.1 \%)$ & $28(25.9 \%)$ \\
\hline \multicolumn{2}{|c}{ Table 2. Histopathology - Epidermis } \\
\hline \multicolumn{2}{|c}{} \\
\hline
\end{tabular}

\section{Correlation with Clinical Diagnosis}

Out of the 217 cases in which a clinical or differential diagnosis of psoriasis were entertained 180 cases were histopathologically proven as psoriasis. It was seen that 64 (36 $\%$ ) out of the 180 cases had a primary clinical diagnosis of psoriasis. In the rest of the cases psoriasis was included only as differential diagnosis. Few of the consistence findings in my study were hyperkeratosis, parakeratosis, regular acanthosis and dilated blood vessels. Munro's micro abscess, even though a characteristic feature of psoriasis, was observed only in 41 $(38 \%)$ of the cases.

\section{DISCUSSION}

\section{Age}

Mean age of the patients in this study was 44 years. The most common age group of patients was between 31.25 and 55.75 years. The mean age among males was $44.2 \mathrm{yrs}$. and for that of females was 43.7 yrs. In the study conducted by Okhandiar et al, highest incidence was noted in $20-39$ yrs. \& mean age of males \& females were comparable.

\section{Gender}

There were 80 males and 28 females in our study with male to female ratio of 2.9:1. In the study by Kaur et al, Bedi et al, Nikhil Moorchung et al and Okhandiar et al were 2:1, 2.5:1, 1.2:1, $2.46: 1$, respectively.

\section{Disease Duration}

In our study, the disease duration ranged from 4 months -40 years with a mean duration of 6.69 years. In a study by Cemal Bilac, Aylin Turel Ermertcan et al, the duration ranged from 1 month -40 months with a mean of $12.4 \pm 9.9$ months. ${ }^{5}$

\section{Comorbidities}

In several studies it has been found that psoriasis is associated with a number of behavioural and systemic comorbidities like obesity, hypertension, diabetes, hyperlipidemia, metabolic syndrome, cardiovascular diseases etc. As such it is important to screen for these diseases among psoriasis patients and give prompt treatment. 6 in my study, there were 16 patients with diabetes mellitus, 23 with hypertension, 9 had both diabetes mellitus and hypertension, and 7 patients gave history of cardiovascular diseases.

\section{Clinical Features}

The major clinical features of psoriasis are erythematous scaly plaques, scalp scaling, pruritus, psoriatic arthritis and nail changes which includes onycholysis and subungual hyperkeratosis. The most frequent clinical findings in the present study was erythematous scaly plaques $(73 \%)$. There were pustules in $14.8 \%$ and generalized exfoliation in $16.6 \%$ of cases, both directing towards diagnosis of variants of psoriasis (Table. 4). Clinical Features observed by Bedi et al ${ }^{7}$ and present study when compared Erythematous scaly plaques (90\%) present study (73\%). Scalp scaling (62\%) 
present study (39.8) Nail changes (54\%) present study (49\%) Pruritus (88 \%), present study (58.3 \%) Palm \& sole involvement (7\%), present study (21.3\%) Psoriatic arthritis (1.3\%) present study (4.6\%).

\section{Histopathology}

Histopathological features pertaining to psoriasis were studied in detail. The cardinal histopathological features of psoriasis include a combination of the following: confluent parakeratosis, regular acanthosis, scattered mitosis of basal and prickle cells, dilatation and tortuosity of dermal capillaries and mild perivascular infiltration with lymphocytes. All the characteristic feature may not be present in one section alone. 8

\section{Epidermal Changes}

The epidermal changes described were hyperkeratosis, confluent parakeratosis and regular epidermal hyperplasia which is known as psoriasiform hyperplasia/ skin reaction pattern. Hyperkeratosis could be identified in 108 (100\%) and is one of the commonest histological features described in psoriasis. However, in a study by Puri et al hyperkeratosis was noted in only $64 \%$ of the cases. Parakeratosis was found in 106 $(98.1 \%)$ of the cases. Confluent parakeratosis helps differentiate psoriasis from other psoriasiform reactions like pityriasis rubra pilaris. ${ }^{47,48}$ Among these $57.4 \%$ were confluent parakeratosis and the rest (40.7\%) were focal. In a study done by Nikhil Moorchung, JS Khullar, Manas Chatterjee, Biju Vasudevan et al, the degree of hyperkeratosis showed a strong correlation with parakeratosis. ${ }^{9}$

Papillomatosis, the surface elevation caused by the hyperplastic epithelium is characteristic of the variant called verrucous psoriasis. ${ }^{10}$ it was seen only in 6 cases (5.55\%) and was mild, none of which warranted the special categorization. Regular acanthosis was seen in 79 (73.1\%) of cases. The remainder of $26.9 \%$ of cases had irregular acanthosis. Saw toothed rete ridges forming irregular acanthosis is characteristically described in lichen simplex chronicus and lichen planus. This finding in our study could be due to eczematisation or chronic itching. ${ }^{11}$

Hypogranulosis, is due to the increased cell turnover, so that granular layer is thinned out in favour of the para/hyperkeratotic layers. This feature was seen in 95 (87.9 $\%)$ of cases. The rest $13(12 \%)$ did not show marked hypogranulosis. This may be due to treatment effect or due to resolution.

Suprapapillary thinning, another feature representing increased turnover was observed in $83(76.9 \%)$ of cases. Similarly, mitotic figures were seen in $80(74.1 \%)$ of cases. The cases with absence of suprapapillary thinning, hypogranulosis and mitosis, probably represents resolving phases of the lesion. $12,13,14$

Still other epidermal features include spongiosis with neutrophilic exocytosis and Munro's micro abscess formation. Spongiosis was seen in 83 (76.9\%) of cases. Psoriasis is primarily spongiotic and it is seen in early lesions and certain specific sites, but it never forms spongiotic vesicles as in eczematous dermatitis. The diagnosis of eczematous dermatitis is preferred over psoriasis when spongiosis is marked, but some cases have been diagnosed as psoriasis with eczematization, since the epidermal hyperplasia and type of inflammatory cell infiltrate favour the latter. ${ }^{15,1}$

Exocytosis of neutrophils was present in 66 cases (61.1\%). Munro microabscess, could be identified in 41(38\%). In the study by Puri, Munro's micro abscess was seen in $30 \%$ of cases. Still rarer is the collection in the spinous layer, called the pustules of Kogoj; which was seen in only $5.6 \%$ of cases. 16,17 Puri $\mathrm{N}$ et al tabulated that there is a reduction in spongiosis and Munro's micro abscess in skin biopsies post treatment. Chronic plaque type psoriasis denotes stable lesions of trunk and extremities. ${ }^{18}$ seven such cases were noted during the study. Pustular psoriasis is a rare, acute form of psoriasiform dermatosis characterized by the widespread eruption of numerous sterile pustules on an erythematous base. 4 (3.7\%) of such cases were detected which correlated with a clinical diagnosis of generalized pustular psoriasis. 19,20

\section{Dermal Changes}

Dermis is affected earlier than epidermis in psoriasis. The earliest histopathological change is the dilatation and congestion of vessels in the papillary dermis and a mild, perivascular, lymphocytic infiltrate, with some adjacent edema. ${ }^{21}$ In the present study dilated blood vessels were seen in around 105 (97.2\%) of cases accounting for the most consistent feature, following hyperkeratosis. Study by Puri et al showed comparable results.

\section{Upper Dermal Infiltrates}

The most common are neutrophils and lymphocytes. ${ }^{22}$ The present study confirmed the same with lymphocytes in $64.8 \%$ and mixed inflammatory infiltrate in the rest. Guttate psoriasis is an early lesion of psoriasis in which, the superficial dermal edema and perivascular infiltrate can be correlated with the typical clinical features. ${ }^{23}$ two such cases were obtained during the study period. Follicular psoriasis is characterized by follicular plugging, marked parakeratosis of ostium and perifollicular and perivascular inflammatory infiltrate. ${ }^{1}$ two such cases were identified in the present study.

\section{Correlation with Clinical Diagnosis}

Dermatology is a field with immense need for clinicopathological correlation. In the present study, cases diagnosed as psoriasis histopathologically were included. On comparison, it was seen that $38(36 \%)$ of the 108 cases had psoriasis as the primary clinical diagnosis. In the rest of the cases, psoriasis was included only in the differential diagnosis.

Clinically florid cases show characteristic erythema and silvery white scales with Auspitz sign. Atypical or involuting or healed lesions pose diagnostic dilemma to the clinician. Although there is a need for clinical data for histological diagnosis of psoriasis, there are definite histopathological features which separate psoriasis from other papulosquamous diseases. Few of the consistent finding in the study were hyperkeratosis, parakeratosis, regular acanthosis and dilated blood vessels. 24,25 Munro's micro abscess, even though considered to be a distinct feature may be explained by the constant exfoliation of corneal layer. Some of the disparities reflected in the histopathological picture may be due to biopsies taken from quiescent lesions. 


\section{Variants}

There are different clinical variants for psoriasis, including sebopsoriasis, flexural, guttate, erythrodermic and pustular psoriasis. Histopathologically psoriasis vulgaris accounted for 93 (86 \%) of the cases. The variants encountered in the study were Exfoliative psoriasis, Pustular Psoriasis, Guttate psoriasis and Follicular psoriasis (Table 4). According a study by Bedi chronic plaque type psoriasis was the most common (90\%) clinical phenotype. 26

Four cases of Generalised pustular psoriasis diagnosed histologically by the presence of spongiform pustules of Kogoj; and two cases of guttate psoriasis, by dermal edema is recorded in this study. The importance of clinical data in these cases cannot be overemphasized.

\section{CONCLUSIONS}

Psoriasiform lesions appear morphologically similar to the prototypical classical psoriasis. Depending upon the disorder, lesions vary in size, shape, scaling and configuration. However, at times, these lesions pose diagnostic dilemma to the treating clinician. The low incidence of its diagnosis and precise assessment, histopathological evaluation is essential. Moreover, in the context of treatment, it is important to differentiate between the various subtypes of psoriasis. It also has an important role in the follow up of these patients.

Financial or Other Competing Interests: None.

\section{REFERENCES}

[1] Weedon D. Weedons' skin pathology. $3^{\text {rd }}$ edn. Churchill Livingstone 2009:72-91.

[2] Elder DE, Elenitsas R. Lever's histo pathology of skin. 10 ${ }^{\text {th }}$ edn. Lippincott Williams and Wilkins 2008:174-81.

[3] Mehta S, Singal A, Singh N, et al. A study of clinichistopathological correlation in patients of psoriasis and psoriasiform dermatitis. Indian J Dermatol Venereol Leprol 2009;75(1):100.

[4] James WD, Berger TG. Andrews' diseases of the skin: clinical dermatology. $10^{\text {th }}$ edn. Elsevier Health Sciences 2006:193-201.

[5] Bilac C, Ermertcan AT, Bilac DB, et al. The relationship between symptoms and patient characteristics among psoriasis patients. Indian J Dermatol Venereol Leprol 2009;75(5):551.

[6] Guenther L, Gulliver W. Psoriasis comorbidities. J Catan Med Surg 2009;13 Suppl 2:S77-87.

[7] Bedi TR. Clinical profile of psoriasis in North India. Indian J Dermatol Venereol Leprol 1995;61(4):202-5.
[8] Puri N, Mahajan BB, Kaur S. Clinicohistopathological correlation of psoriasis in acute exacerbation. Open Access Sci Rep 2012;1(9):455.

[9] Moorchung N, Khullar JS, Mani NS, et al. A study of various histopathological features and their relevance in pathogenesis of psoriasis. Indian J Dermatol 2013;58(4):294-8.

[10] Khalil FK, Keehn CA, Saeed S, et al. Verrucous psoriasis: a distinctive clinicopathologic variant of psoriasis. Am J Dermatopathol 2005;27(3):204-7.

[11] Marks R, Wells GC. Lichen simplex: morphodynamic correlates. Br J Dermatol 1973;88(3):249-56.

[12] Gunasti S, Marakli SS, Tuncer I, et al. Clinical and histopathological findings of 'psoriatic neurodermatitis' and of typical lichen simplex chronicus. J Eur Acad Dermatol Venereol 2007;21(6):811-7.

[13] Ragaz A, Ackerman AB. Evolution, mutation, and regression of lesions of psoriasis. New observations and correlation of clinical and histologic findings. Am J Dermatopathol 1979;1(3):199-214.

[14] Gordon M, Johnson WC. Histopathological and histochemistry of psoriasis. I. The active lesion and clinically normal skin. Arch Dermatol 1967;95(4):402-7.

[15] Stadler R, Schaumberg-Lever G, Orfanos CE. Histology. In: Mier PD, van de Kerkhof PCM, ed. Textbook of psoriasis. Edinburgh: Churchill Livingstone 1986:40-54.

[16] Cribir BJ. Psoriasis under the microscope. J Eur Acad Dermatol Venereol 2006;20(Suppl 2):3-9.

[17] Van de Kerkhof PCM. Neutrophils in psoriasis: cause or effect? Clin Exp Dermatol 2000;25(2):165.

[18] Burns T, Breathnach S, Cox N, et al. Rook's text book of dermatology. $8^{\text {th }}$ edn. Wiley-Blackwell 2013:20.5-20.7.

[19] Shelley WB, Kirschbaum JO. Generalized pustular psoriasis. Arch Dermatol 1961;84(1):123-8.

[20] Kingery FA, Chinn HD, Saunders TS. Generalized pustular psoriasis. Arch Dermatol 1961;84:912-9.

[21] Chowaniec 0, Jablonska S, Beutner EH, et al. Earliest clinical and histological changes in psoriasis. Dermatologica 1981;163(1):42-51.

[22] Krueger JG, Bowcock A. Psoriasis pathophysiology: current concepts of pathogenesis. Ann Rheum Dis 2005;64(Suppl 2):ii30-6.

[23] Barr RJ, Young EM. Psoriasitorm and related papulosquamous disorders. J Cutan Pathol 1985;12(5):412-25.

[24] Boyd AS, Menter A. Erythrodermic psoriasis. precipitating factors, course and prognosis in 50 patients. J Am Acad Dermatol 1989;21(5 Pt 1):985-91.

[25] Prystowsky JH, Cohen PR. Pustular and erythrodermic psoriasis. Dermatol Clin 1995;13(4):757-70.

[26] Dogra S, Yadav S. Psoriasis in India: prevalence and pattern. Indian J Dermatol Venereol Leprol 2010;76(6):595-601. 